\title{
REFEREES 2017
}

\section{AGRADECIMENTO AOS REVISORES EM 2017}

A Finisterra agradece a todas as pessoas que participaram nas tarefas de revisão e avaliação do conteúdo científico dos textos submetidos para publicação.

O sucesso da Finisterra depende do entusiasmo, voluntarismo e apoio contínuos do seu painel de peritos e peritas. Mas também dos autores e autoras por acolherem as apreciações acerca dos seus manuscritos com a elevação que o espírito científico solicita.

Para além dos/as revisores/as que manifestaram interesse em manter o total anonimato e por isso os seus nomes não são aqui divulgados, a Finisterra destaca os/as colegas que anonimamente avaliaram os manuscritos em 2017:

\section{THANKS TO THE REFEREES 2017}

The Editor thanks all the referees for their participation in the review process of the texts submitted for publication.

The success of the journal depends on the continued enthusiasm, willingness and support of this panel of experts. But also of the authors for embracing peer reviews with an open scientific spirit.

In addition to the reviewers who have expressed an interest in maintaining total anonymity and therefore their names are not disclosed here, Finisterra highlights the colleagues who blind reviewed the manuscripts in 2017:

\begin{tabular}{|c|c|}
\hline Alcides Goularti Filho & Guilhem Mousselin \\
\hline Alina Esteves & Héctor Mendoza Vargas \\
\hline Andy Inch & Helena Esteves \\
\hline Àngel Cebollada Frontera & Herculano Cachinho \\
\hline Ángel Miramontes Carballada & Hugo Arruda de Morais \\
\hline Ángel Pueyo Campos & Isabel Salamaña i Serra \\
\hline Antoni Tulla & Jennifer McGarrigle Carvalho \\
\hline Antonio Cezar Leal & Jesús Monteagudo López-Menchero \\
\hline Aureanice Corrêa & João Araújo-Gomes \\
\hline Brenda Matossian & João Cabral \\
\hline Carlos Ferrás Sexto & João de Abreu e Silva \\
\hline Carlos Neto & João Guerreiro \\
\hline Carlos Schaefer & João Jarochinski Silva \\
\hline César Magalhães & João Rafael Santos \\
\hline Cian O> Callaghan & Joaquín Farinós Dasí \\
\hline Cristina Henriques & Jorge Alberto Sanchez Espinosa \\
\hline Dámaris Padilha & Jorge Gonçalves \\
\hline David Vale & Jorge Malheiros \\
\hline Denis Linehan & Jorge Rocha \\
\hline Denise Bomtempo & Jorge Trindade \\
\hline Diogo Abreu & Jorge Umbelino \\
\hline Duval Fernandes & José Afonso Teixeira \\
\hline Eda Góes & José Alberto Lima de Carvalho \\
\hline Eduarda Marques da Costa & José Alberto Rio Fernandes \\
\hline Eduardo Ascensão & José Carlos Ferreira \\
\hline Eduardo Brito-Henriques & José Gaspar \\
\hline Eduardo Castro & José Gomes dos Santos \\
\hline Eduardo Souza de Morais & José Manuel Simões \\
\hline Elisa Pinheiro de Freitas & José Ramiro Pimenta \\
\hline Elsa Pacheco & Luís Carvalho \\
\hline Enric Mendizabal & Luís Junqueira \\
\hline Eusébio Reis & Luiz Antonio Machado da Silva \\
\hline Fátima Matos & Manuel Castelo Branco \\
\hline Fernando Teigão dos Santos & Marcelo Fragoso \\
\hline Francisco Roque de Oliveira & Márcio Cataia \\
\hline Frederico Moura e Sá & Margarida Pereira \\
\hline Gerry O'Reilly & Margarida Queirós \\
\hline Gilberto Rocha & Maria Fernanda Alegria \\
\hline Gonçalo Vieira & Maria Filomena Silvano \\
\hline
\end{tabular}

\author{
Maria João Alcoforado \\ Maria José Roxo \\ Maria Laura Silveira \\ Miguel Padeiro \\ Nelson Mileu \\ Niamh Moore-Cherry \\ Nuno Costa \\ Nuno Ganho \\ Patrícia Abrantes \\ Patrícia Marques \\ Paulo Morgado \\ Paulo Roberto Baqueiro Brandão \\ Pedro Arsénio \\ Rafael Gotardi Brússolo \\ Rafaello Bergonse \\ Ricardo Garcia \\ Rita Raposo \\ Rui Gama \\ Rui Pedro Julião \\ Sandra Oliveira \\ Sérgio Oliveira \\ Simon Sanches Moral \\ Simone Preuss \\ Simone Tulumello \\ Sónia Pereira \\ Susana Sassone \\ Susana Veleda da Silva \\ Sylvio Fausto Gil Filho \\ Sylwia Kaczmarek \\ Teresa Barata Salgueiro \\ Teresa Cabrita \\ Tito Machado de Oliveira \\ Vanice Selva \\ Vasco Reis \\ Victor Ribeiro Filho \\ Yuri Simonini \\ Zeny Rosendahl
}




\section{AGRADECIMENTO AOS MEMBROS DO JÚRI DO MELHOR ARTIGO DE 2016}

A Finisterra agradece o apoio científico, a participação nas discussões e a disponibilidade para avaliar os conteúdos da revista ao Júri do Prémio Artigo do Ano 2016, composto por:
THANKS TO THE JURY OF THE BEST SCIENTIFIC PAPER 2016

Finisterra thanks the scientific support, the participation in the jury discussions and the willingness of the Jury to evaluate the contents of the journal for the Prize of the Article of the Year 2016, composed by:

\author{
Maria João Alcoforado (Presidente | President) \\ Helena Esteves \\ Jorge Malheiros \\ José Carlos Teixeira \\ Joseli Maria Silva \\ Paulo Morgado
}

Em 2016, o Artigo do Ano da Finisterra foi atribuído, ex-aequo a:

Simone Tulumello e Marco Picone, "Shopping malls and neoliberal trends in Southern European cities: post-metropolitan challenges for urban planning policy", Finisterra - Revista Portuguesa de Geografia, LI(101): 111-132;

Pedro Porfírio Coutinho Guimarães, "A eficácia dos projectos especiais de urbanismo comercial. Evidências de Braga", Finisterra - Revista Portuguesa de Geografia, LI(102): 47-64.

Menção Honrosa do Júri:

Anna Ortiz e Cristóbal Mendoza, “Jóvenes doctorandos europeos y latinoamericanos en Barcelona: experiencias migratorias y vivencias en la ciudad", Finisterra - Revista Portuguesa de Geografia, LI(102): 81-101.
In 2016, the Article of the Year, was awarded ex-aequo to:

Simone Tulumello and Marco Picone, "Shopping malls and neoliberal trends in Southern European cities: post-metropolitan challenges for urban planning policy", Finisterra - Revista Portuguesa de Geografia, LI(101): 111-132;

Pedro Porfírio Coutinho Guimarães, "The effectiveness of the special projects of commercial urbanism: Evidence from Braga", Finisterra - Revista Portuguesa de Geografia, LI(102): 47-64.

Honorable Mention by the Jury:

Anna Ortiz and Cristóbal Mendoza, "Young Phd students from Europe and Latin America in Barcelona: migration experiences and everyday practices in the city", Finisterra - Revista Portuguesa de Geografia, LI(102): 81-101, was honored with an.

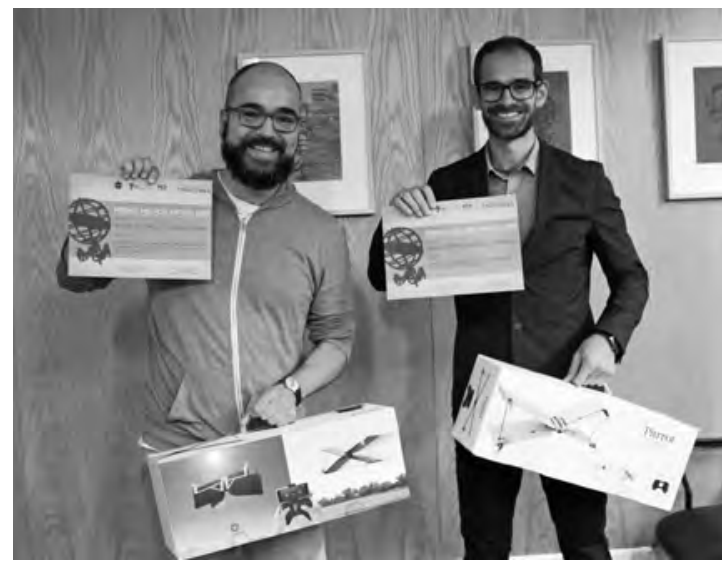

Simone Tulumello; Pedro Guimarães

Cerimónia de atribuição do Prémio Artigo do Ano 2016 | Award Ceremony Article of the Year 2016

A cerimónia de entrega do Prémio Artigo do Ano 2016 associou-se à Lição Anual da Finisterra 2017

The Award of the 2016 Article of the Year was held together with the Annual Lecture of the Finisterra 2017 